\title{
Nucleation and growth of carbon nanotubes by microwave plasma chemical vapor deposition
}

\author{
Chris Bower ${ }^{\text {a) }}$ and Otto Zhou ${ }^{\text {b) }}$ \\ Department of Physics and Astronomy, University of North Carolina, Chapel Hill, North Carolina 27599
}

Wei Zhu, D. J. Werder, and Sungho Jin

Bell Laboratories, Lucent Technologies, Murray Hill, New Jersey 07974

(Received 23 May 2000; accepted for publication 29 August 2000)

\begin{abstract}
The nucleation and growth of aligned multiwall carbon nanotubes by microwave plasma-enhanced chemical vapor deposition have been studied. The nanotubes nucleate and grow from catalytic cobalt islands on a silicon substrate surface, with both their diameter and length dependent on the size of the cobalt islands. Electron microscopy reveals that the nanotubes grow via a "base growth" mechanism. The nanotubes grow initially at a very rapid and constant rate $(\sim 100 \mathrm{~nm} / \mathrm{s})$ that decreases sharply after the catalyst Co particles become fully encapsulated by the nanotubes. We propose a detailed model to explain these experimental observations on nucleation and growth of nanotubes. (C) 2000 American Institute of Physics. [S0003-6951(00)00443-5]
\end{abstract}

Carbon nanotubes with different structure and morphology can now be fabricated with several techniques. Experiments performed, mostly on individual nanotubes, show that they have extraordinary electrical and mechanical properties. ${ }^{1}$ To investigate their macroscopic properties and potential technological applications it is essential to have nanotube materials with controlled diameter, length, orientation, location, and microstructure. Although the laser ablation method $^{2}$ can produce high quality single-walled carbon nanotubes with some control in diameter and electronic properties, ${ }^{3}$ the process is not compatible with conditions required for device fabrication.

Considerable progress has been made in fabrication of aligned multiwalled nanotubes (MWNTs) by chemical vapor deposition (CVD) methods. ${ }^{4-7}$ Although these processes differ in growth conditions such as substrate, catalyst, feed gas, and temperature, the growth model proposed by several groups essentially adopts the established concepts of CVD carbon fiber growth developed in the $1970 \mathrm{~s} .{ }^{8,9}$ It is believed that nanotubes grow as carbon precipitates from a supersaturated metal catalyst that resides at either the base (i.e., "base growth") or the tip of a growing nanotube (i.e., "tip growth"). Catalyst-substrate interactions and temperature gradients across the catalyst particle are considered to be important factors that determine the growth mechanism. However, most of these models were proposed without sufficient and systematic supporting experimental evidence, and they often lacked details about the physical mechanisms and the effects of various process parameters. In addition, the kinetics of nanotube nucleation and growth is largely unknown.

We have previously reported the growth of aligned MWNTs using microwave plasma-enhanced CVD. ${ }^{10}$ Here we present a mechanistic study of the nanotube nucleation and growth and the kinetics involved in the microwave plasma CVD process. We found that the nanotubes grow via

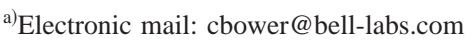

${ }^{b)}$ Curriculum in Applied and Materials Sciences.
}

a "base growth" mechanism in our CVD system, and there is a strong correlation between the catalyst metal layer thickness and the nanotube diameter. We observed that the process kinetics involved a very rapid initial growth followed by a saturation period in which nanotube growth appears to be stunted. We propose a four-step model of nanotube nucleation and growth to explain the experimental data.

The nanotubes were grown in a microwave plasma CVD system connsisting of a $2.45 \mathrm{GHz} 5 \mathrm{~kW}$ microwave power supply and an inductively heated substrate stage. Prior to growth, a thin film $(2-60 \mathrm{~nm})$ of metal catalyst (cobalt) was sputter deposited onto a silicon (100) substrate. Scanning electron microscopy (SEM) showed that the sputtered cobalt films were continuous with only a few pinholes. The samples were transferred (in air) to the growth chamber and heated to $825^{\circ} \mathrm{C}$ in flowing hydrogen $(200 \mathrm{sccm}, 20$ Torr). A $1 \mathrm{~kW}$ microwave plasma was subsequently ignited. At this time, ammonia $\left(\mathrm{NH}_{3}\right)$ was introduced to completely replace the hydrogen, and acetylene $\left(\mathrm{C}_{2} \mathrm{H}_{2}\right)$ was added shortly after $(\sim 0-5 \mathrm{~min})$ to start the nucleation and growth of nanotubes.

Typically, a deposited film could be visually observed 5-10 s after introduction of the acetylene. The carbon nanotube films were dull black in color and could be grown uniformly over 4 in. silicon wafers. Figure 1(a) shows a crosssectional SEM image of a highly oriented MWNT film deposited for 3 min with an initial cobalt layer of $2 \mathrm{~nm}$. This nanotube film was grown at $825^{\circ} \mathrm{C}$ and 20 Torr with a 3 to 1 $(150: 50 \mathrm{sccm})$ ammonia to acetylene ratio. Higher magnifi-

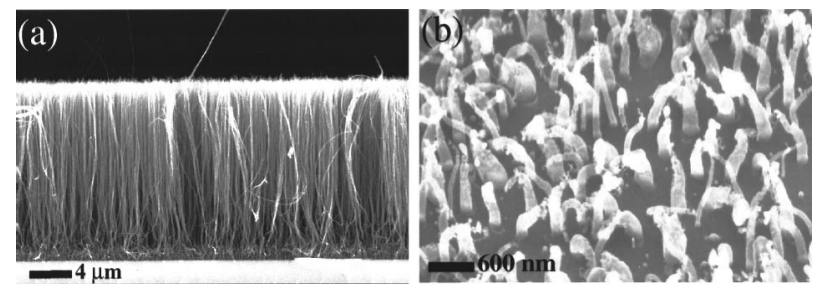

FIG. 1. (a) Aligned 30-nm-diameter MWNTs grown on cobalt coated silicon. The initial cobalt layer thickness was $2 \mathrm{~nm}$. (b) MWNTs grown when the initial cobalt layer thickness was $20 \mathrm{~nm}$. 

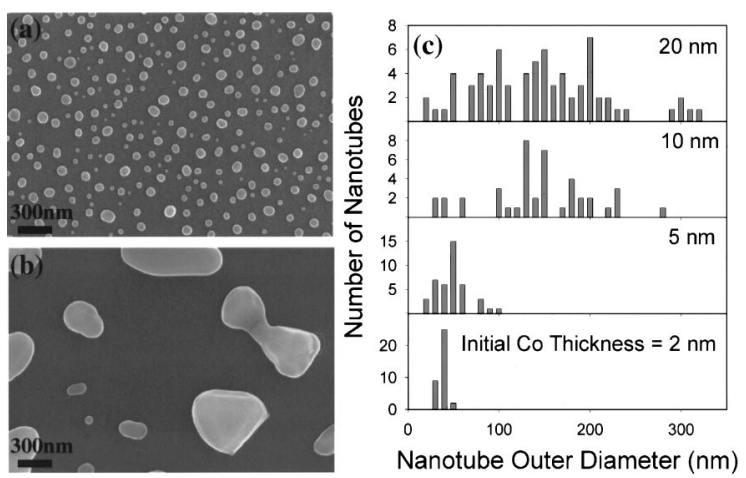

FIG. 2. SEM images of the Co islands which formed from (a) 2-nm-thick and (b) 20-nm-thick Co layers. (c) A histogram showing the distribution of nanotube diameters found with different cobalt layer thickness.

cation SEM and transmission electron microscopy (TEM) studies showed that the nanotubes had a narrow diameter distribution around $30 \mathrm{~nm}$ and were about $12 \mu \mathrm{m}$ in length. For comparison, Fig. 1(b) shows a MWNT film grown for 10 min with an initial cobalt film thickness of $20 \mathrm{~nm}$. The nanotubes deposited here had larger diameters, shorter lengths, and were substantially less uniform in orientation, compared to the nanotubes deposited on the $2 \mathrm{~nm}$ cobalt film shown in Fig. 1(a).

The initially continuous sputter-deposited cobalt film was seen, prior to the nanotube growth, to transform into three-dimensional islands during the temperature ramp-up heating process. The size of the Co islands was dependent on the initial thickness of the sputtered Co, as shown in Figs. 2(a) and 2(b). Thicker cobalt films (>10 nm) yielded larger Co islands on average with a broader size distribution, while thinner cobalt films $(<10 \mathrm{~nm})$ resulted in smaller Co islands on average with a much narrower size distribution. The observed sizes of Co islands and their distributions prior to the nanotube growth were consistent with the nanotube diameters and their distributions after the growth, as shown in Fig. 2(c). The nanotubes produced using $2 \mathrm{~nm}$ of sputtered Co had smaller average diameters of nanotubes and a very narrow diameter distribution centered around $30 \mathrm{~nm}$, while the nanotubes grown on the $20 \mathrm{~nm}$ sputtered Co had a much larger average diameter and a wide diameter distribution. This result suggests that smaller diameter nanotubes, perhaps even single-walled nanotubes, could be produced if smaller catalytic metal islands can be formed.

TEM, performed on nanotubes that had been scraped from the substrate and then briefly ultrasonicated in methanol, showed that the nanotubes were typically composed of 20-40 concentric graphitic shells. Many of the nanotubes $(\geqslant 50 \%)$ had "bamboo", type defects ${ }^{11,12}$ along a part of their length. Cone-shaped Co particles were found enclosed within the ends of the nanotubes, as shown in Fig. 3(a). SEM performed on a sample grown for only $10 \mathrm{~s}$ [inset in Fig. 3(a)] showed that the Co particles were located at the base of the nanotubes. Cross-sectional TEM, shown in Fig. 3(b), also confirmed the presence of the Co particles at the base of the nanotubes, and no Co was ever observed at the nanotube tips. This is strong evidence for a "base growth" mechanism, similar to that reported by Baker and Harris for CVD growth of carbon fibers. ${ }^{8}$ This "base growth" mechanism was further supported by the experimental evidence, pre-
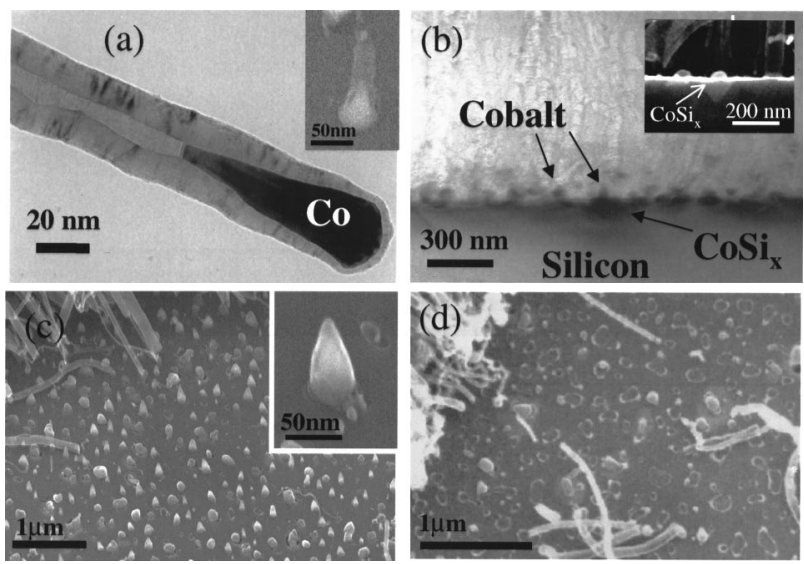

FIG. 3. (a) TEM micrograph of a cone-shaped cobalt particle fully enclosed within one end of the MWNT. The inset shows a nanotube after $10 \mathrm{~s}$ of growth. (b) A cross-sectional TEM image of an aligned MWNT film. The inset shows a cross-sectional SEM image of an aligned MWNT film. (c) A SEM image of the "scratched"' surface of a nanotube film grown for $1 \mathrm{~min}$. The inset is a close-up of one of the cone-shaped cobalt particles left of the surface. (d) A SEM image of the "scratched" surface of a nanotube film grown for $10 \mathrm{~min}$.

sented in our earlier report, ${ }^{10}$ that a growth process consisting of an initial plasma deposition followed by a thermal deposition yielded a straight-to-curly nanotube transition, which occurred at the base. Cobalt silicide $\left(\mathrm{CoSi}_{x}\right)$ was observed by TEM and cross-sectional SEM at the siliconcobalt interface [see the inset of Fig. 3(b)], indicating that the cobalt reacts with the silicon during the process. This reaction might increase the adhesion between the metal catalyst and the substrate, which could explain why the metal catalyst stays at the base of the nanotube during growth.

A growth rate curve, shown in Fig. 4, was obtained by measuring the height of nanotubes grown for differing amounts of time (10 s-30 min), under otherwise identical conditions. The curve shows a very short incubation period $(<10 \mathrm{~s})$ followed by a period $(2-3 \mathrm{~min})$ of rapid and constant growth, which is then followed by a sharp decrease in the growth rate. During the rapid growth, the nanotubes grew at a rate of $100 \mathrm{~nm} / \mathrm{s}$. This is roughly 30 times higher than the growth rate observed with a thermal CVD process. ${ }^{10}$

The decrease in growth rate is attributed to the fact that the catalytic Co particles became encapsulated at the bottom of the nanotubes after an extended period of growth. It was noticed that nanotubes deposited for a short period of time (e.g., less than $3 \mathrm{~min}$ ) do not contain Co particles within their shells at the bottom ends when observed by TEM; rather, the cone-shaped cobalt remained on the substrate surface after the nanotubes were removed, as shown in Fig. 3(c). In contrast, crater shaped marks were left behind on the substrate surface after longer-growth nanotubes (i.e., after the decrease in the growth rate occurred) were removed [see Fig. 3(d)], indicating that the Co particles were enclosed at the nanotube ends. We postulate that the decrease in the growth rate occurs as a result of the cobalt particle becoming fully enclosed within the carbon layers [such as shown in Fig. 3(a)]. Consequently, it became more difficult for the reactive carbon species to reach the catalyst particle at the root of the nanotube and continue the growth process through a presumed diffusion and precipitation process.

The nanotube growth rate was also found to be inversely 


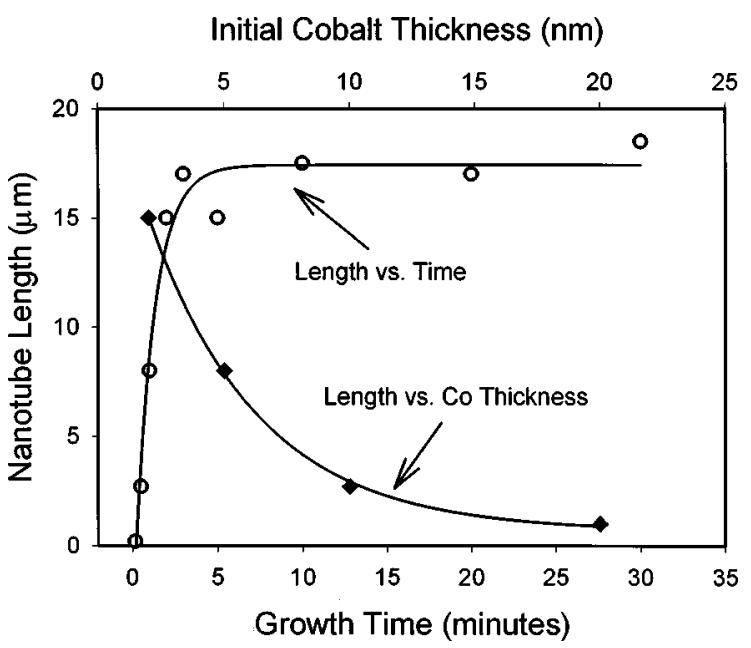

FIG. 4. (a) A plot of the nanotube length vs growth time (O) and a plot of the nanotube length vs initial cobalt film thickness $(\diamond)$. The growth rate data $(\bigcirc)$ were obtained from samples grown on 2-nm-thick Co films and at $\mathrm{T}=825^{\circ} \mathrm{C}$ and $\mathrm{P}=20$ Torr, and with a $3: 1$ ammonia to acetylene ratio. The Co film thickness data $(\checkmark$ ) were obtained from four samples of varying Co thickness grown at the same time under identical conditions.

proportional to the nanotube diameter. Figure 4 shows the average nanotube height measured for four films that were grown simultaneously under identical conditions, with the only variable being the initial thickness of the cobalt film. Note that the cobalt film thickness determines the cobalt island size, which in turn determine the nanotube diameter. Clearly, the smaller diameter nanotubes grew at a faster rate in their height, compared to the larger diameter tubes. These results seem to suggest a constant mass deposition rate of carbon for each nanotube, and this constant rate of mass deposition determines the corresponding height that a nanotube can reach associated with its diameter.

We have developed a step model for nanotube nucleation and growth in our microwave plasma CVD system, as

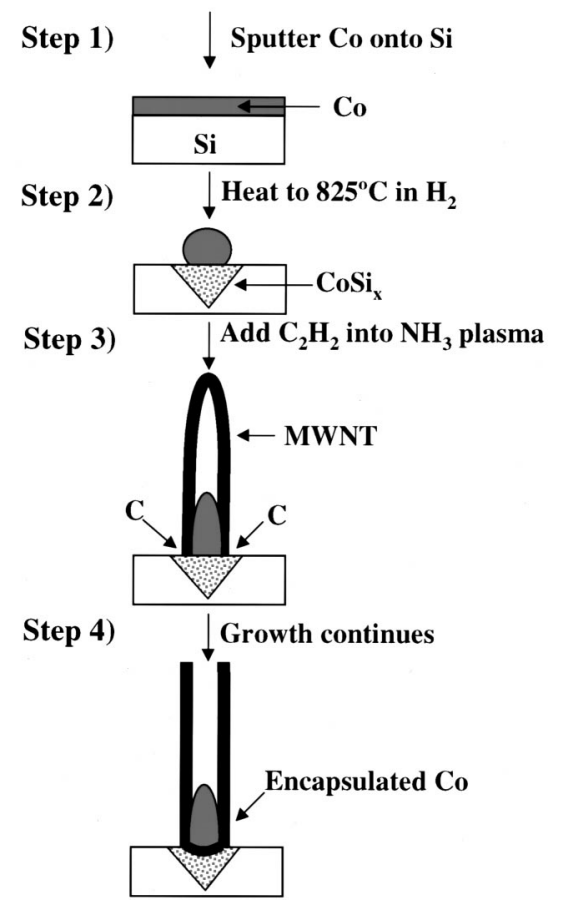

FIG. 5. Schematic of the proposed four-step growth model. schematically illustrated in Fig. 5. The model takes into account the formation of catalytic cobalt islands, the chemical reactions between cobalt and $\mathrm{Si}$ substrate, and the base growth mechanism involved. It also identifies the determining factors for controlling both the nanotube diameter and length. In step 1, a layer of Co is deposited on a Si substrate. In step 2, the Co film on the surface starts to form semispherical islands during heating, driven by surface tension to lower the total energies. These three-dimensional islands provide the nucleation and growth sites for nanotubes, and the size of these islands dictate the diameter of nanotubes. Cobalt silicides are formed at the $\mathrm{Co}-\mathrm{Si}$ interface that consume a portion of the metallic Co and also serve as anchors or adhesion promoters for the Co islands. These silicides are seen by cross-sectional SEM and TEM to be in epitaxial relationship with the Si substrate. During the initial growth stage in step 3, nanotubes nucleate and grow from the cobalt islands with plasma-induced alignment, ${ }^{10}$ likely through carbon reactions with cobalt (dissolution, saturation, and precipitation). The cobalt islands transform into a conical shape and are confined at the bottom ends of the tubes. The growth of nanotubes continues in length until the conical cobalt particles are completely enclosed by the nanotube shells, as illustrated in step 4. At this time, the growth slows down dramatically or possibly completely stops.

In summary, we have presented a mechanistic study of the nucleation and growth of aligned nanotubes by microwave plasma CVD. We found that the initial thickness of the catalytic Co layer or the size of the Co islands essentially dictates the nanotube diameters, and that the catalyst islands remain at the base of the nanotube throughout the growth process. We found that nanotubes grow initially at a very rapid rate followed by a dramatic decrease in its growth, apparently after the catalytic particles are fully enclosed by the nanotube shells.

The authors acknowledge R. Tung and A. G. Ramirez for useful discussions. C.B. acknowledges fellowship support from NASA. O.Z. acknowledges funding support from ONR MURI Grant No. N00014-98-1-0597.

${ }^{1}$ M. S. Dresselhaus, G. Dresselhaus, and P. C. Eklund, Science of Fullerenes and Carbon Nanotubes (Academic, New York, 1996).

${ }^{2}$ A. Thess, R. Lee, P. Nikolaev, H. Dai, P. Petit, J. Robert, C. Xu, Y. H. Lee, S. G. Kim, A. G. Rinzler, D. T. Colbert, G. E. Scuseria, D. Tomanek, J. E. Fischer, and R. E. Smalley, Science 273, 483 (1996).

${ }^{3}$ X.-P. Tang, K. A. H. Shimoda, L. Fleming, K. Y. Bennoune, C. Bower, O. Zhou, and Y. Wu, Science 288, 492 (2000).

${ }^{4}$ W. Z. Li, S. S. Xie, L. X. Qian, B. H. Chang, B. S. Zou, W. Y. Zhou, R. A. Zhao, and G. Wang, Science 274, 1701 (1996).

${ }^{5}$ Z. F. Ren, Z. P. Huang, J. W. Xu, J. H. Wang, P. Bush, M. P. Siegal, and P. N. Provencio, Science 282, 1105 (1998).

${ }^{6}$ S. Fan, M. G. Chapline, N. R. Franklin, T. W. Tombler, A. M. Cassell, and H. Dai, Science 283, 512 (1999).

${ }^{7}$ J. Li, C. Papadopoulos, J. M. Xu, and M. Moskovits, Appl. Phys. Lett. 75, 367 (1999).

${ }^{8}$ R. T. K. Baker and P. S. Harris, in Chemistry and Physics of Carbon, edited by P. L. Walker and P. A. Thrower (Marcel Dekker, New York, 1978), Vol. 14.

${ }^{9}$ G. G. Tibbetts, J. Cryst. Growth 66, 632 (1984).

${ }^{10}$ C. Bower, W. Zhu, S. Jin, and O. Zhou, Appl. Phys. Lett. 77, 830 (2000).

${ }^{11}$ Y. Saito, in Carbon Nanotubes, edited by M. Endo, S. Ijima, and M. S. Dresselhaus (Elsevier, Oxford, 1996), p. 159.

${ }^{12}$ H. Cui, D. Palmer, O. Zhou, and B. R. Stoner, in Amorphous and Nanostructured Carbon, edited by J. Sullivan, J. Robertson, O. Zhou, T. Allen, and B. Coll [Mater. Res. Soc. Symp. Proc. 593, 39 (1999)]. 